\title{
Pairing of Pentagonal and Heptagonal Carbon Rings in the Growth of Nanosize Carbon Spheres Synthesized by a Mixed-Valent Oxide-Catalytic Carbonization Process
}

\author{
Z. L. Wang* and Z. C. Kang \\ School of Material Science and Engineering, Georgia Institute of Technology, Atlanta, Georgia 30332-0245
}

Received: August 29, $1996^{\otimes}$

\begin{abstract}
Carbon spheres have been synthesized using a mixed-valent oxide-catalytic carbonization process in macroscopic quantities and at low cost. The technique uses natural gas and reusable catalysts and produces no environmental waste or pollution. The product is all solid spheres $(\sim 210 \mathrm{~nm})$ comprised of layered graphitic flakes. Microstructures of the spheres are studied by transmission electron microscopy. The sphere is believed to be nucleated from a pentagon carbon ring followed by a spiral shell growth. When the sphere grows larger, graphitic flakes are nucleated on the surface due to the formation of paired pentagonal-heptagonal $(\mathrm{P}-\mathrm{H})$ carbon rings. A combination of the $\mathrm{P}-\mathrm{H}$ pairs with the hexagonal networks produces eight basic graphitic configurations for forming the sphere, and they have been observed experimentally.
\end{abstract}

\section{Introduction}

Carbon is a versatile element because it can form various structures. ${ }^{1,2}$ The successful growth of diamond films, ${ }^{3-5}$ the fullerene molecule $\mathrm{C}_{60}{ }^{6}$ and its family $\mathrm{C}_{n}$, ${ }^{7,8}$ and carbon nanotubes has excited many researchers. ${ }^{9-11}$ The variety of structures produced by carbon are determined by its unique hybridization $\mathrm{sp}^{1}, \mathrm{sp}^{2}$, and $\mathrm{sp}^{3}$ bonding. One of the most striking contributions made by the fullerene and carbon nanotubes ${ }^{12}$ is that the carbon atoms can form pentagonal and heptagonal carbon rings, and the combination of the two basic structural units with the hexagonal carbon rings can form a variety of geometrical configurations. ${ }^{13}$ Fullerene carbon spheres were observed by Iijima ${ }^{14}$ using a high-resolution transmission electron microscope (HRTEM). Carbon spheres with diameter of a few nanometers were obtained in HRTEM by irradiating a soot containing tubular and polyhedral graphitic particles using an intense high-energy electron beam. ${ }^{15}$ Heat treatment of pure carbon soot ${ }^{16}$ and ultradispersed diamond powders $(2-6 \mathrm{~nm})^{17}$ also produced small size carbon spheres. These small size carbon spheres are so-called carbon "onions". Carbon spheres with sizes of $1-10 \mu \mathrm{m}$ were found in carbon black produced by carbonization of polyethylene-poly(vinylchloride) in a sealed gold tube ${ }^{18}$ under a pressure of $30 \mathrm{MPa}$ and in carbon vapor produced by decomposition of $\beta$-SiC powder. ${ }^{19}$

In this paper, a new technique is described for synthesizing monodispersed high-percentage (>95\%), solid carbon spheres (or tubes) in macroscopic quantities at very low cost. ${ }^{20}$ The growth mechanism of the graphitic carbon spheres is proposed using the microstructural information provided by HRTEM. Various structural configurations of the graphitic flakes, due to the presence of hexagonal, pentagonal, and heptagonal carbon rings, are proposed. It is concluded that the pairing of pentagonal and heptagonal carbon rings is the key for growing large size carbon spheres.

\section{Synthesis of Carbon Spheres}

The synthesis uses natural gas (methane) as the source of carbon. Natural gas flowed through a quartz tube, in which

\footnotetext{
* Fax: (404) 894-9140. E-mail: zhong.wang@mse.gatech.edu.

${ }^{\otimes}$ Abstract published in Advance ACS Abstracts, October 15, 1996.
}

catalytic transition and/or rare earth metal oxides with mixed valences had been placed. Decomposition of $\mathrm{CH}_{4}$ occurred when the chamber was heated to $1100{ }^{\circ} \mathrm{C}$, and carbon spheres/ tubes were formed on the wall of the chamber. The process was controlled by temperature to produce either all carbon spheres at $1100{ }^{\circ} \mathrm{C}$ or all pure carbon tubes at $950{ }^{\circ} \mathrm{C}$. The product was separated from the catalysts in the growth chamber, and no capsulation was found. The unreacted methane gas can be recycled, and no environmental waste and pollution will be produced. This technique is referred to as a mixed-valent oxidecatalytic carbonization (MVOCC) process. ${ }^{20,21}$ In this paper, we concentrate on the studies of carbon spheres, and the results on carbon spiral tubes are reported separately. ${ }^{22}$

Under heating, the metal oxide releases lattice oxygen due to the change in the density of the cation element with different valence states in the compound. The released atomic oxygen on the catalyst surface oxidizes $\mathrm{CH}_{4}$ and takes away the hydrogen atoms to form water vapor, resulting in the formation of carbon rings. The oxide catalysts are still free standing and nondestructive after the reaction. After the growth the catalysts can be retreated in oxygen/air to regain oxygen and reused. Using the oxides as catalysts is the key in MVOCC. The success of using mixed-valence metal oxides as catalysts has opened a new field in catalysis research and applications.

Scanning electron microscopy (SEM) images were recorded using a Hitachi S800 SEM. HRTEM studies were performed at $400 \mathrm{kV}$ using a JEOL 4000EX TEM.

\section{Microstructure of the Carbon Spheres}

Figure 1a is a typical SEM image of the monodispersive, high-percentage carbon spheres, as indicated by the size distribution plot given in Figure 1b. The carbon spheres have an average diameter of $\sim 210 \mathrm{~nm}$, much smaller than those found in carbon black. ${ }^{18}$ More than $85 \%$ of the carbon spheres have almost the same size. The spherical shape is best seen in the TEM image (Figure 2a). The spheres are dispersive without aggregation.

Figure 2a,b shows a pair of bright-field and dark-field TEM images of the synthesized nanosize carbon spheres. The carbon spheres are perfectly round, smooth, and clean. The spheres are solid and do not contain any large porosity. The cone shape contrast is due to the curvature of the bent (001) graphitic planes 

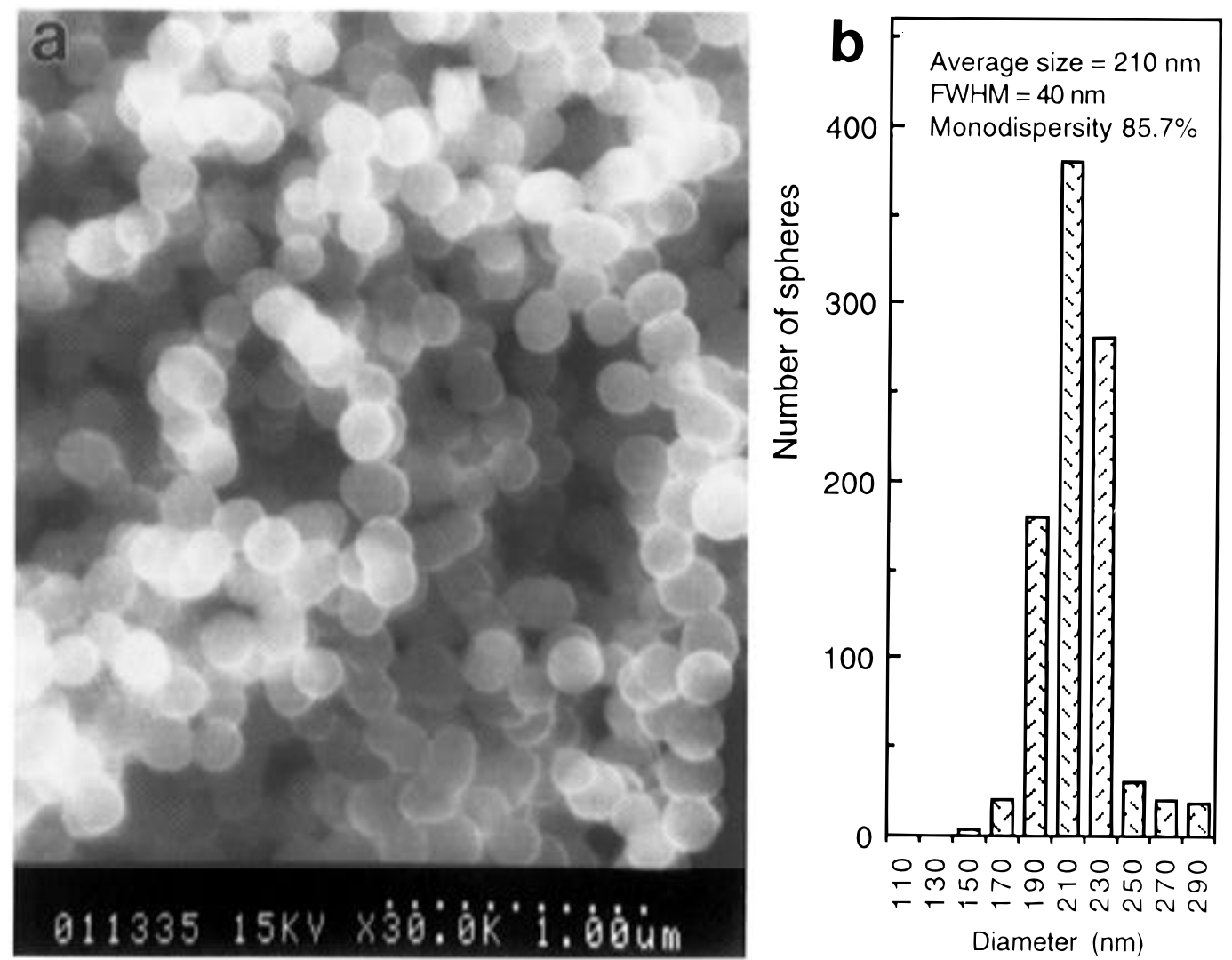

Figure 1. (a) SEM image of carbon spheres synthesized at $1100{ }^{\circ} \mathrm{C}$, showing monodispersive size distribution as indicated by the plot in (b). The product is pure, and no carbon tubes or any other product was observed. The image was recorded at $15 \mathrm{kV}$ using a Hitachi S800 SEM.
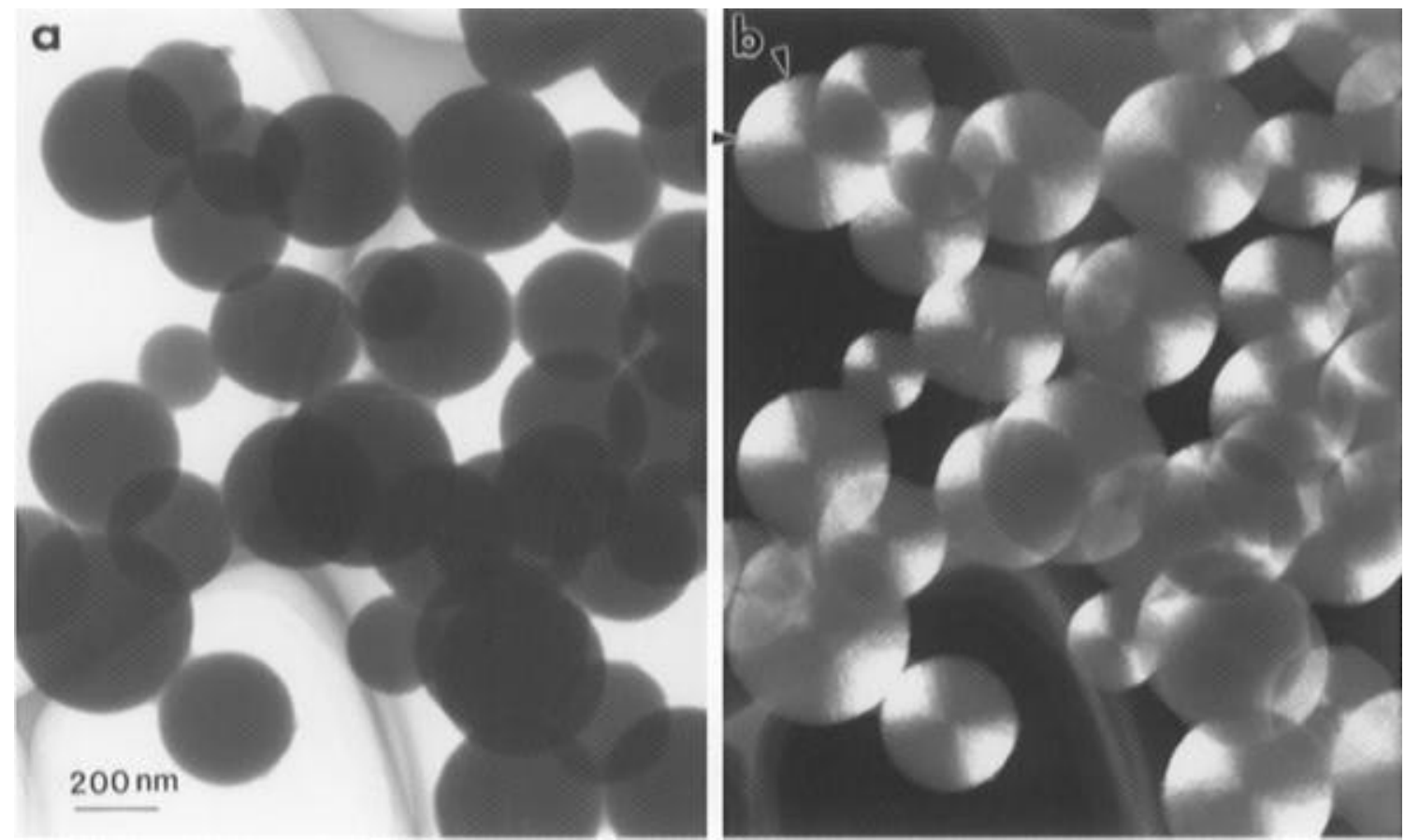

Figure 2. (a) Bright-field and (b) dark-field TEM images of the synthesized carbon spheres. The spheres are solid, clean, smooth, and without porosity. The dark-field image is recorded using part of the $\{101\}$ reflection ring.

following the curvature of the sphere and the finite size of the objective aperture in TEM. With a detection limit of $1 \%$, energy dispersive X-ray spectroscopy in TEM indicates that the spheres are made of carbon.

The spheres are composed of $c$-axis-oriented graphitic layers, and the piling up of these flat layers follows the curvature of the inner layers from the center of the sphere to the external surface (Figure 3), but each layer is not a closed shell. The graphitic flakes are in the size range of $1-30 \mathrm{~nm}$, and they are arranged with a partial order.

The spheres are solid. With the use of a $400 \mathrm{kV}$ TEM, we are able to image the microstructure near the center of the spheres (Figure 4). It must be pointed out that the graphitic layers that are parallel to the incident beam are dramatically decreased upon approaching the center, because we are imaging the sphere along a tangential direction. Considering the 


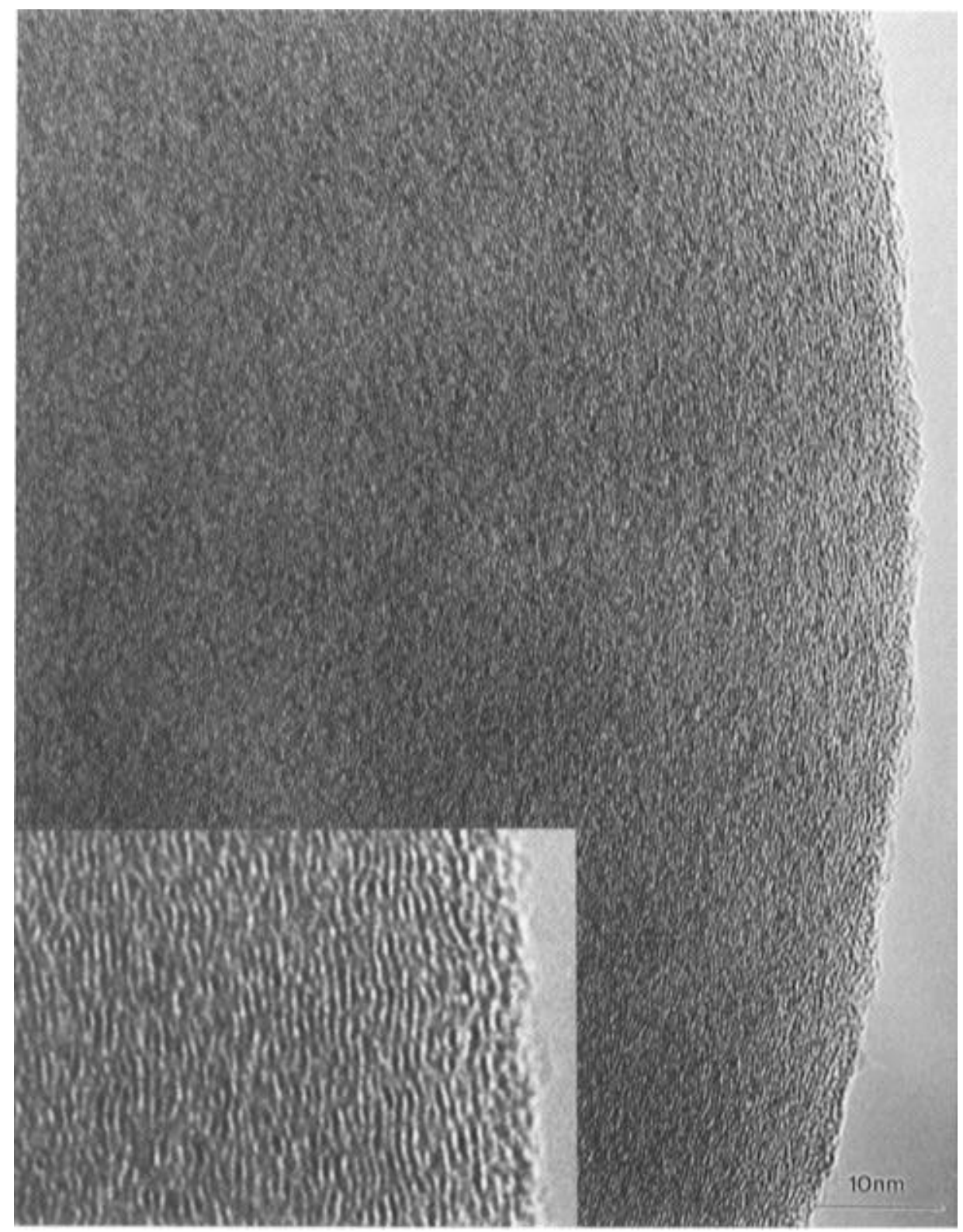

Figure 3. HRTEM image of a carbon sphere showing that the sphere is composed of a piling up of graphitic layers, with the $c$-axis approximately parallel to the radial direction. The inset is a magnified region.

influence of the large thickness of the sphere near the center, the image contrast is relatively poor at the center, but this does not indicate that the center part is disordered or amorphous.

\section{Pairing of Pentagonal and Heptagonal Carbon Rings}

In this section, we examine the details of the image contrast. Figure 5 shows two high-magnification TEM images of the carbon spheres. The sphere is composed of curling graphitic flakes exhibiting waving structure. When the electron beam is positioned at the edge of the sphere (Figure 5c), the diffraction pattern shows a pair of arc shape reflections with $d=0.34 \pm$ $0.02 \mathrm{~nm}$, corresponding approximately to the (002) interlayer distance of graphite. The presence of the (002) reflection shows that the (001) plane is nearly parallel to the electron beam in this case. The arc shape is due to the curvature of the sphere covered by the electron beam. The continuous rings of $\{101)$ and $\{112\}$, with $d=0.2$ and $d=0.12 \mathrm{~nm}$, respectively, indicate the random twist among (001) graphitic layers. When the electron beam is positioned at the center of the sphere (Figure $5 d)$, the diffraction pattern shows only the $\{101)$ and $\{112\}$ rings. The absence of the (002) reflection indicates that the local (001) graphite plane is nearly perpendicular to the electron beam. The circle in Figure 5c indicates the position of the objective aperture in TEM used to record the dark-field image shown in Figure 2b. The finite size of the aperture picks up only the reflections generated by the curling graphitic flakes falling within the cone region, as indicated by the arrowheads in Figure 2b, resulting in the cone shape contrast of the spheres. Therefore, the spheres are composed of curling graphite flakes with random twist. The graphitic layers forming the carbon spheres are not closed shells but small size waving flakes, ranging in size from 1 to $30 \mathrm{~nm}$ and covering the spherical surface. From the HRTEM images, a total of eight different basic structural configurations have been found, as indicated by the model numbers in Figures $5 \mathrm{a}, \mathrm{b}$ and 6 . The waving follows certain patterns determined by the atom arrangements in the graphitic flakes, as described in the following.

To form a graphitic carbon sphere, the atomic structure of each flake must be one of the known graphite carbon rings, and the graphitic lattice must be modulated to accommodate the curvature of the sphere. The carbon atoms can form three different types of graphitic carbon rings (Figure 7). The flat 


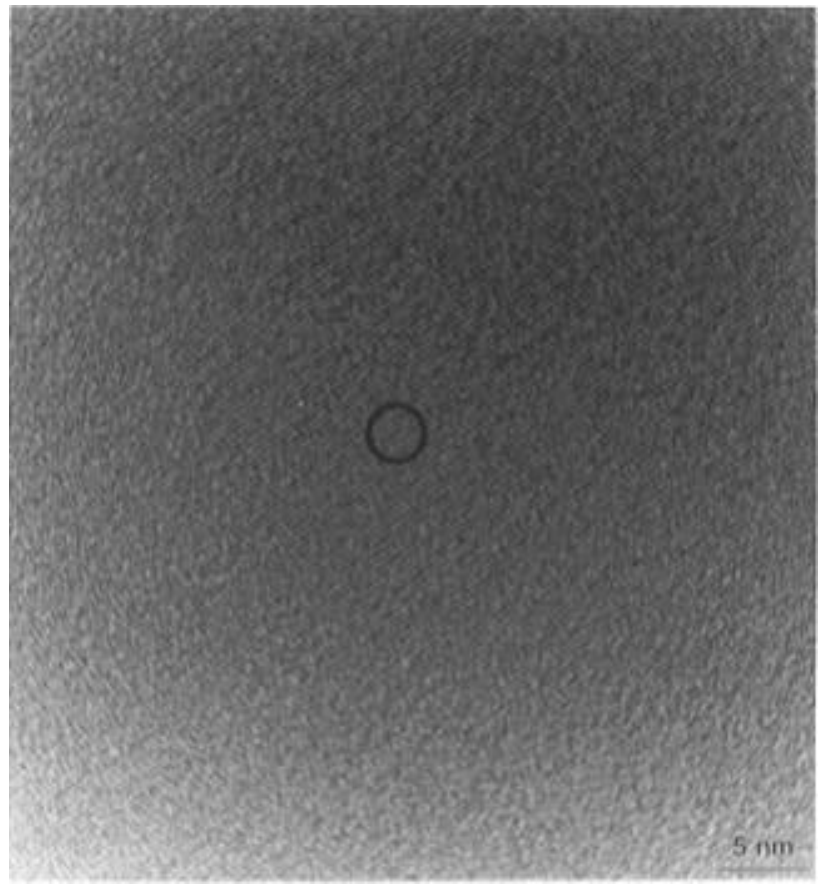

Figure 4. HRTEM image of a carbon sphere recorded near the center showing that the sphere is solid. The graphitic flakes around the center are imaged.

graphite layer is composed of hexagonal carbon rings (Figure 7a). A pentagonal carbon ring (Figure 7b) causes the hexagonal network to curve inward (with $+60^{\circ}$ disclinations), forming a surface with positive curvature. The heptagonal carbon ring forces the hexagonal lattice to be curved outward (with $-60^{\circ}$ disclinations). ${ }^{12}$ The curving produced by a heptagon is opposite to that by a pentagon. Thus, if a pair of pentagon and heptagon appears in the hexagonal lattice, the curving of the entire lattice would be dramatically reduced.

A pure hexagonal network cannot form a perfectly closed shell. Euler's theorem shows that a perfectly closed shell can be formed if exactly 12 pentagonal carbon rings are introduced in the hexagonal network, although the number of hexagons is arbitrary. Thus, pentagonal carbon- rings are essential for forming closed carbon cage structures, such as $\mathrm{C}_{60}$. However, a $\mathrm{C}_{60}$ is unlikely to be the nucleation site of a carbon sphere because of its closed structure. A curling graphitic carbon particle is believed to be nucleated from a pentagonal carbon ring, the growth of which gives a quasi-icosahedral spiral shell carbon particle (Figure 8). This is the nucleation mechanism proposed by Kroto and $\mathrm{McKay}^{7}$ for the formation of large fullurenes. A pentagonal carbon ring produces an inward surface with positive curvature, resulting in a spiral growth. At the beginning, the particle appears in a quasi-icosahedral shape. ${ }^{7}$ When the particle size is much larger than the interlayer spacing $d(=0.34 \mathrm{~nm})$, the particle is approximately a sphere. However, this single spiral shell carbon sphere cannot continuously grow along the spiral direction for the following reason. With the increase of the particle surface area, the newly created pentagons and heptagons in the reaction chamber fall continuously on the surface of the sphere. These pentagons are new nucleation sites for growing curved graphitic flakes with orientations that may not have any relation to the initial spiral layer. In this case, the growth rates of these graphitic flakes might be much faster than that of the spiral layer. With the formation of many pentagonal and heptagonal carbon rings, the particle surface is covered by a stack of randomly twisted graphitic flakes nucleated at different depth (or time), consistent with the observations shown in Figure 5.
A spherical particle cannot be formed if the heptagons are absent, because pentagonal carbon rings will lead to the growth of a large faceted polyhedron. Therefore, the pentagons need to be paired with the heptagons in order to nucleate a nonfaceted graphitic layer that follows the topology of the inner layers, provided the sizes of the graphitic layers can be changed. The two adjacent pentagon and heptagon should be unstable and tend to recombine to form two hexagonal carbon rings. However, when the pentagon and heptagon are interconnected by hexagons, various structural configurations can be formed, as shown in Figure 9. The experimental images of these structural configurations are indicated in Figures 5 and 6 by the model numbers. Since one carbon atom may not have more than four nearest neighbors, a carbon atom located at the edges of the flakes cannot be simultaneously shared by three graphitic flakes. Thus, one graphitic flake is not directly linked to the corner of a heptagonal carbon ring, resulting in a small gap in models 4 and 8 (see Figure 9). The experimental image shown in Figure $5 \mathrm{a}$ (see the areas indicated by 4 and 8 ) clearly indicates the existence of this gap. Model 3 is frequently observed in the images, as shown in the circled areas in Figure $5 \mathrm{~b}$ and in Figure $6 \mathrm{~b}, \mathrm{c}$, corresponding to a stacking of graphitic flakes with pentagon-on-pentagon and heptagon-on-heptagon structures. The density of this type of structural configuration increases significantly after the specimen is annealed in air at $1100{ }^{\circ} \mathrm{C}$ for $24 \mathrm{~h}$, indicating the lower energy state of this configuration in comparison to others. Using the eight structural models, the geometrical shapes of all the observed graphitic flakes in the HRTEM images can be qualitatively constructed if the size of the graphitic flake can be changed. The most frequently observed structural configurations are 2, 3, 4, 5, and 8. The presence of numerous pentagon-heptagon $(\mathrm{P}-\mathrm{H})$ pairs can significantly modulate the interlayer distance, resulting in the broadening of the (002) peak (Figure 5c).

Geometrically, a surface cannot be perfectly closed if the number of pentagons equals that of the heptagons. ${ }^{23}$ Therefore, the surface must be composed of unclosed graphitic flakes with sizes determined by the growth rate. For spheres with diameters of less than $40 \mathrm{~nm},{ }^{15}$ the growth is probably dominated by the spiral growth. ${ }^{7}$ Therefore, a large graphitic carbon sphere, excluding the central core with spiral structure, is composed of graphitic flakes containing the $\mathrm{P}-\mathrm{H}$ pairs. The formation of the $\mathrm{P}-\mathrm{H}$ pairs is determined by the synthesis technique and experimental conditions. If the experimental conditions are changed so that the creation rates and fractions of pentagons and heptagons are fluctuated, the carbon particles grow into different geometrical shapes, such as spiral tubes with nodes. ${ }^{22}$

\section{Discussion}

Carbon spheres with closed-shell "onion" structure produced under the radiation of a high-energy electron beam might have been considered as the "ideal" model of carbon spheres. Unfortunately, these spheres cannot be produced in macroscopic quantities. The spheres synthesized using MVOCC have a monosize distribution and are in macroscopic quantities. The average size of approximately $210 \mathrm{~nm}$ is ideally suited for many practical applications in advanced technology and engineering. In fact, the carbon onions are not expected to be formed in the MVOCC process because of high nucleation rates of the paired $\mathrm{P}-\mathrm{H}$ carbon rings. To form the carbon onion structure, no nucleation of heptagonal carbon rings is allowed, and the nucleation rate of the pentagonal carbon rings must be precisely controlled to be 12 pentagons per shell. This is experimentally extremely difficult. In addition, the carbon onions may not grow into large spheres because the atom species must be deposited 

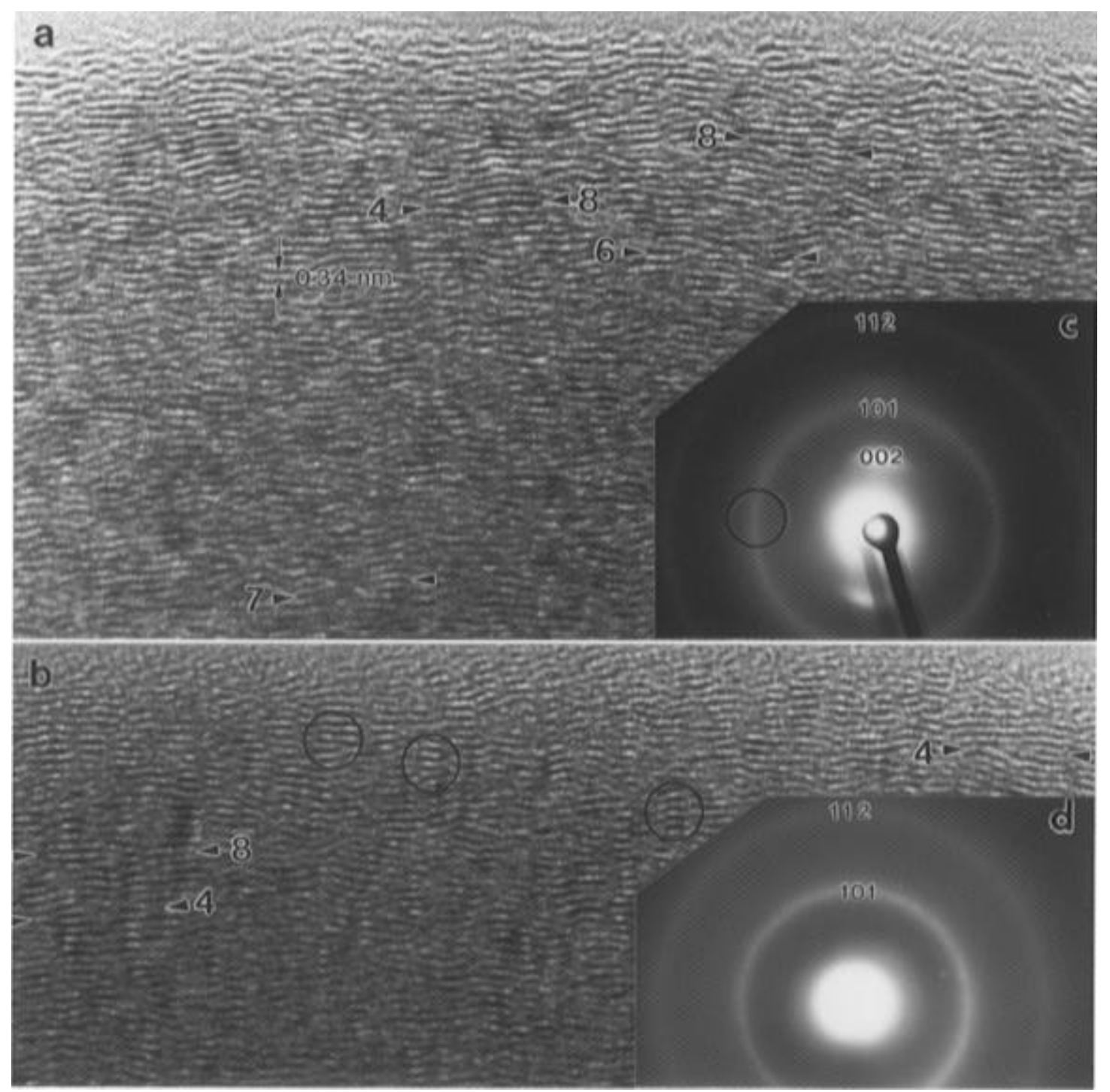

Figure 5. (a, b) HRTEM images of the spherical carbon particles near the edges, showing the graphitic waving structures. The sequence numbers appearing in the areas indicated by the paired arrowheads represent the structural models described in Figure 9 . The interlayer distance is $0.34 \mathrm{~nm}$. $(c, d)$ Electron diffraction patterns recorded from a carbon sphere when the electron probe is positioned at the edge and the center of the sphere, respectively.

at the edge of the in-plane growth front. Therefore, the graphitic flake structure is the model for growing large spheres. A postgrowth annealing at $2000{ }^{\circ} \mathrm{C}$ may dramatically improve the shell-like structure of the spheres.

The carbon spheres produced with MVOCC are not the same as the spheres found in carbon black for the following reasons. First, the MVOCC process is a new technique that is substantially different from the techniques ${ }^{19,20}$ which have been demonstrated to produce carbon black particles. Our spheres were synthesized at a moderate temperature $\left(1100{ }^{\circ} \mathrm{C}\right)$ under atmospheric pressure rather than the $30 \mathrm{MPa}$ high pressure used in ref 19. Second, the carbon spheres produced by the MVOCC are monodispersive with an average size of $210 \mathrm{~nm}$, which is much smaller than the sizes of the spheres found in carbon black $(1-10 \mu \mathrm{m}$ in diameter). The product has a high percentage $(>95 \%)$ of carbon spheres, while the fraction of carbon spheres found in carbon black is very low and their sizes are uncontrollable. Finally, our spheres are solid, and they are nucleated and grown on the basis of the formation of pentagonal and heptagonal carbon rings. However, the carbon black particles were believed to be hollow spherical shells that were composed of piling up of hexagonal graphite flakes without the presence of $\mathrm{P}-\mathrm{H}$ carbon rings. ${ }^{24}$ The structural model of carbon particles found in carbon black was proposed on the basis of dark-field imaging and electron diffraction results. ${ }^{25,26}$ In their model, the sphere was considered to be composed of small aromatic layers which are assumed to be aligned approximately on circular conical surfaces with a common vertex in the spherule at or near the center of the spherule. According to this model, the structure of the sphere has a cylindrical symmetry around the common vertex rather than a spherical symmetry. This model, however, cannot explain the microstructure of our spheres.

The MVOCC process has following advantages in comparison to the other techniques for synthesizing carbon spheres. The fraction of carbon spheres is high, and the product contains a high percentage of spheres $(>95 \%)$; thus, no purification or separation is needed. The product is not encapsulated on the surface of the catalyst, and carbon spheres have a narrow size distribution. The technique allows synthesis of macroscopic quantities of spheres as needed for measurement of their physical properties and practical applications.

\section{Conclusion}

Using a mixed-valent oxide-catalytic carbonization (MVOCC) technique, nanosize carbon spheres are synthesized with highpercentage ( $>95 \%)$, macroscopic quantities of monodispersity. 


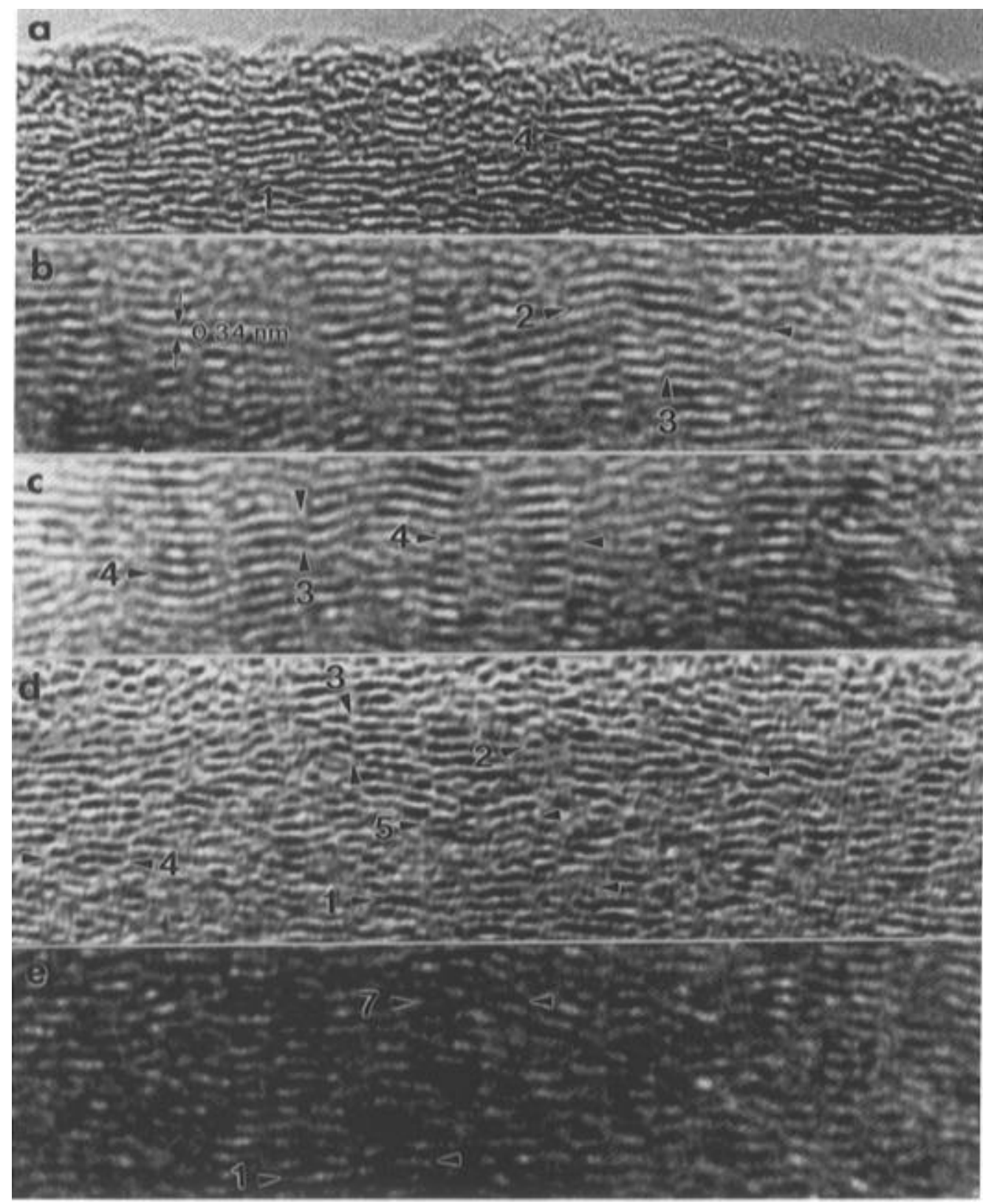

Figure 6. HRTEM images of the spherical carbon particles showing the various types of graphitic waving structures in the areas indicated by arrowheads. The sequence numbers appearing in the areas represent the structural models described in Figure 9 . The interlayer distance is 0.34 nm in each image.

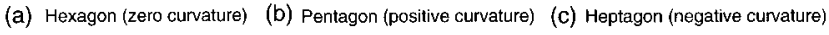
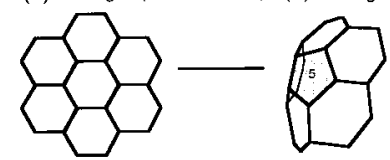

Carbon sphere

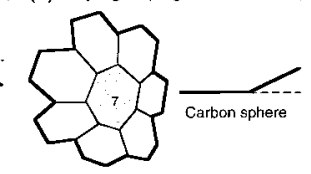

Figure 7. (a) Hexagonal, (b) pentagonal, and (c) heptagonal carbon ring structures in graphitic flakes. The curving of the graphitic flake resulting from these structures is schematically shown using the twodimensional models for the convenience of HRTEM image interpretation, although the curving occurs on a 3-D surface.

The sphere is solid and is believed to be nucleated from a pentagon carbon ring followed by a spiral shell growth. When the particle becomes larger, graphitic segments of atomic thickness are nucleated on the surface due to the arrival of pentagonal-heptagonal $(\mathrm{P}-\mathrm{H})$ carbon rings. The presence of the $\mathrm{P}-\mathrm{H}$ pairs in hexagonal networks is the key for the growth of large spheres. The geometrical configurations of the graphitic flakes can be qualitatively constructed using the combination of pentagonal, heptagonal, and hexagonal carbon rings. The

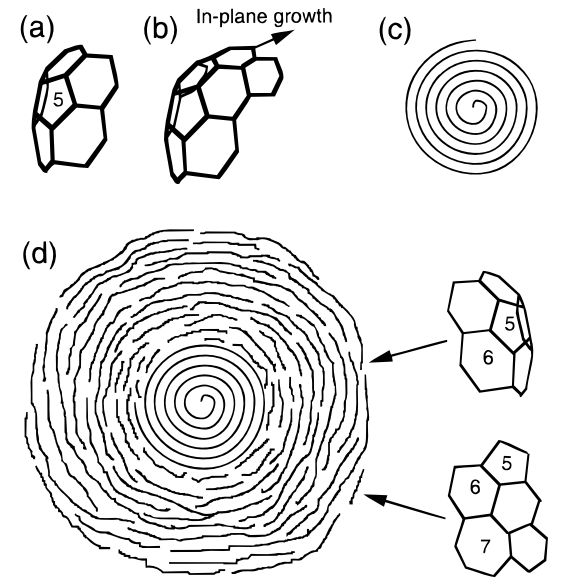

Figure 8. (a) Nucleation of a pentagon, (b) growth of a quasiicosahedral shell, and (c) formation of a spiral shell carbon particle proposed by Kroto and McKay. ${ }^{7}$ (d) Growth of a large size carbon sphere. 


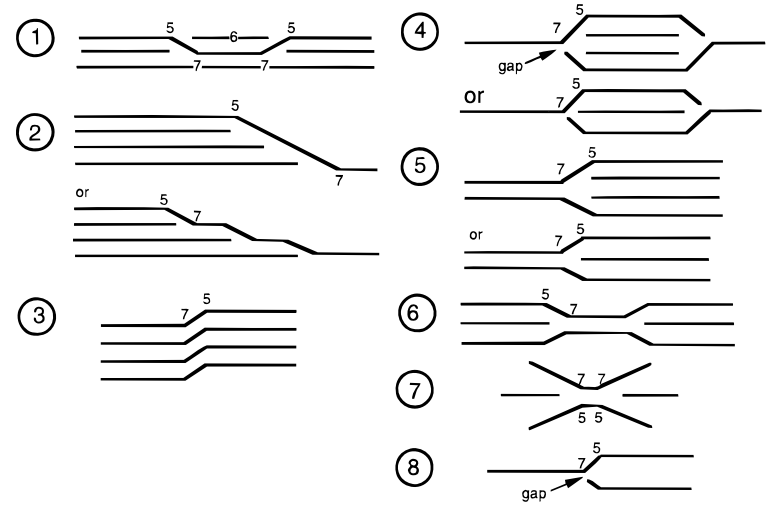

Figure 9. Two-dimensional structural models of graphitic flakes produced by the combination of pentagonal (5), hexagonal (6), and heptagonal (7) carbon rings. The pentagons are paired with heptagons. In these models, the inner graphitic layers are assumed to be located beneath the models. It must be pointed out that the 3-D curving of the graphitic surface is symbolically represented by a 2-D line in order to be compared to the experimental images provided by HRTEM. The experimental images of these models are indicated in Figures 2 and 3 using the same sequence numbers.

variation in size of these graphitic flakes can create any curvature required to meet the geometry of the inner layers.

\section{References and Notes}

(1) Robertson, J. Prog. Solid State Chem. 1991, 21, 199.
(2) Rao, C. N. R.; Seshadri, R.; Govindaraj, A.; Sen, R. Mater. Sci. Eng. 1995, R15, 209.

(3) Angus, J. C.; Hayman, C. C. Science 1988, 241, 913.

(4) Yarbrough, W. A.; Messier, R. Science 1990, 247, 688 181.

(5) Bachmann, P.; Lydtin, H. Mater. Res. Soc. Symp. Proc. 1990, 165,

(6) Kroto, H. W.; Heath, J. R.; O’Brien, S. C.; Curl, R. F.; Smalley, R. E. Nature 1985, 318, 162.

(7) Kroto, H. W.; McKay, K. Nature 1988, 331, 328.

(8) Lamb, L. D.; et al. Science 1992, 255, 1413.

(9) Iijima, S. Nature 1991, 354, 56.

(10) Mintimire, J. W.; Dunlap, B. I.; White, C. T. Phys. Rev. Lett. 1992 $68,63$.

(11) Hamada, N.; Sawada, S.; Oshlyama, A. Phys. Rev. Lett. 1992, 68, 1579.

(12) Iijima, S.; Ichihashi, T.; Ando, Y. Nature 1992, 356, 776.

(13) Ōsawa, E.; Yoshida, M.; Fujita, M. MRS Bull. 1994, 19, 33-36.

(14) Iijima, S. J. Cryst. Growth 1980, 50, 675.

(15) Ugarte, D. Nature 1992, 359, 707.

(16) de Heer, W.; Ugarte, D. Chem. Phys. Lett. 1993, 207, 480.

(17) Kuznetsov, V. L.; Chuvilin, A. L.; Butenko, Y. V.; Mal'kov, I. Y.; Titov, M. Chem. Phys. Lett. 1994, 222, 343.

(18) Inagaki, M.; Kuroda, K.; Sakai, M. Carbon 1983, 21, 231.

(19) Yamada, K.; Tobisawa, S. Carbon 1989, 27, 845.

(20) Kang, Z. C.; Wang, Z. L. U.S. Pat. Appl. 60/008,925, 1996.

(21) Kang, Z. C.; Wang, Z. L. Philos. Mag. 1996, B73, 905.

(22) Wang, Z. L.; Kang, Z. C. Phil. Mag. 1996, B74, 51.

(23) Jones, D. E. H. New Sci. 1966, Nov 3, 245. Jones, D. E. H. The Inventions of Daedalus; Freeman: Oxford, 1982; pp 118-119.

(24) Heidenreich, R. D.; Hess, W. M.; Ban, L. L. J. Appl. Crystallogr. 1968, 1,1 .

(25) Hishiyama, Y.; Yoshida, A.; Inagaki, M. Carbon 1982, 20, 79.

(26) Ayache, J.; Oberlin, A.; Inagaki, M. Carbon 1990, 28, 337.

JP962762F 